\title{
Measurement of Collins asymmetries for kaons and pions at BABAR
}

\author{
Georges Vasseur* ${ }^{*}$ \\ IRFU, CEA, Université Paris-Saclay, \\ F-91191 Gif sur Yvette, France \\ E-mail: georges.vasseur@cea.fr
}

\begin{abstract}
Inclusive hadron production cross sections and angular distributions in $e^{+} e^{-}$collisions shed light on fundamental questions of fragmentation processes. Here are presented measurements of the Collins azimuthal asymmetries in inclusive production of hadron pairs, in the $e^{+} e^{-} \rightarrow h_{1} h_{2} X$ annihilation process, where the two hadrons, $h_{1}$ and $h_{2}$, either kaons or pions, are produced in opposite hemispheres. The data collected by the BABAR detector allow the determination of the Collins fragmentation function dependence on hadron fractional energy for the up, down and, for the first time, strange quarks.
\end{abstract}

38th International Conference on High Energy Physics

3-10 August 2016

Chicago, USA

\footnotetext{
* Speaker.

On behalf of the BABAR collaboration.
} 


\section{Motivation}

Fragmentation, the process through which quarks or gluons hadronize into colourless hadrons, is described via fragmentation functions. Those universal functions contain non-perturbative information and have to be determined by experiments. A fragmentation function gives the probability for a quark to produce a given hadron carrying an energy $E_{h}$ equal to a certain fraction $z=2 E_{h} / \sqrt{s}$ of half the total available energy $\sqrt{s}$.

In case of a transversely polarized quark $q \uparrow$, with spin $s_{q}$ and momentum direction $k_{q}$, fragmenting into a spinless hadron of mass $M_{h}$ and transverse momentum $P_{\perp}$ with respect to $k_{q}$, the fragmentation function can be expessed as:

$$
D_{1}^{q \uparrow}\left(z, P_{\perp} ; s_{q}\right)=D_{1}^{q}\left(z, P_{\perp}\right)+\frac{1}{z M_{h}} H_{1}^{q \uparrow}\left(z, P_{\perp}\right) s_{q} \cdot\left(k_{q} \times P_{\perp}\right) .
$$

It is divided into two parts: an unpolarized fragmentation function $D_{1}^{q}$ and a term dependent on a spin-orbit coupling, expressed through the mixed product $s_{q} \cdot\left(k_{q} \times P_{\perp}\right)$, which leads to an asymmetry in the azimuthal angular distribution of the final states particles around the quark direction [1]. This behaviour is called the Collins effect. The polarized fragmentation function $H_{1}^{q \uparrow}$ present in this term gives the amplitude of the effect. It is also called the Collins fragmentation function.

The Collins effect is an azimuthal modulation, which can be studied either in semi-inclusive deep inelastic scattering, by sending an unpolarized lepton beam on polarized targets, or in $e^{+} e^{-}$ annihilation followed by the hadronization of the produced $q \bar{q}$ pair. In the latter case, the directions of the $q$ spin and $\bar{q}$ spin are unknown, but they have to be the same. Thus by studying the azimuthal correlation between two hadrons $h_{1}$ and $h_{2}$ produced in opposite hemispheres in the $e^{+} e^{-} \rightarrow h_{1} h_{2} X$ inclusive annihilation process, the azimuthal modulation can be observed, which leads to the measurement of the product of two Collins fragmentation functions, one for the fragmenting quark and one for the fragmenting antiquark.

\section{Analysis}

Two different reference frames have been used in the analysis to describe the process. Ideally the direction of the quark-antiquark pair in the centre of mass sholud be used. However, this direction is not known experimentally, but it can be approximated by the thrust axis of the event. In the thrust reference frame shown in figure 1, the scattering plane is defined by the beam axis and the thrust axis, with $\theta_{t h}$ being the angle between the two axes. The modulation of the cross section is a cosine function of the sum of the azimuthal angles $\phi_{1}$ and $\phi_{2}$ of the two hadrons with respect to this plane:

$$
\sigma \sim 1+\frac{\sin ^{2} \theta_{t h}}{1+\cos ^{2} \theta_{t h}} \cos \left(\phi_{1}+\phi_{2}\right) \frac{H_{1}^{\uparrow}\left(z_{1}\right) \bar{H}_{1}^{\uparrow}\left(z_{2}\right)}{D_{1}\left(z_{1}\right) \bar{D}_{1}\left(z_{2}\right)} .
$$

In the other hadron reference frame, one of the two hadrons, for example $h_{2}$, is taken as reference. The scattering plane is defined by the beam axis and the direction of $h_{2}$. The modulation of the cross section is a cosine function of twice the azimuthal angle $\phi_{0}$ of $h_{1}$. The latter reference frame is simpler from an experimental point of view. However it leads to a more difficult theoretical 


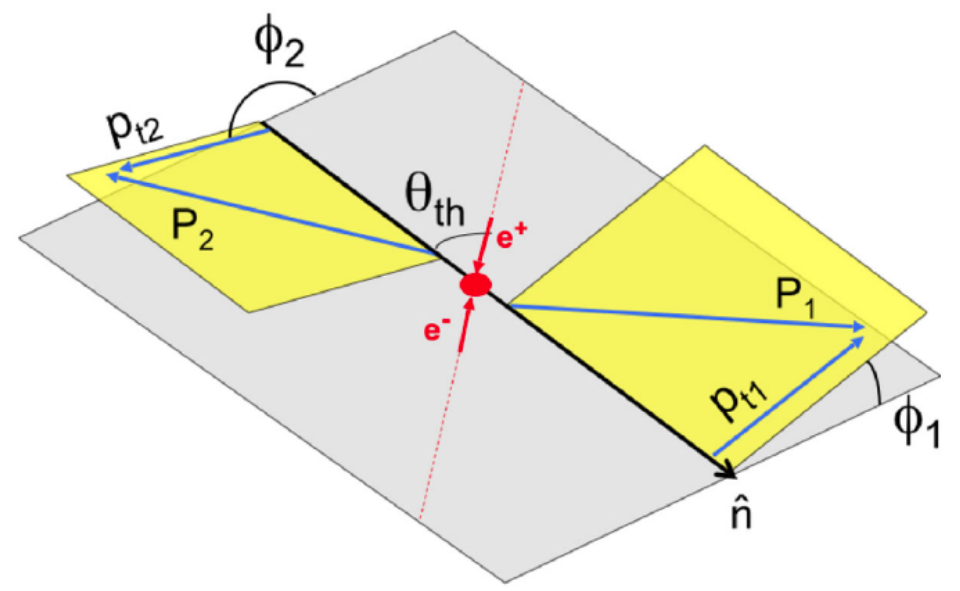

Figure 1: Definition in the thrust reference frame of the azimuthal angles $\phi_{1}$ and $\phi_{2}$ of the two hadrons as the angles between the scattering plane and their transverse momenta $p_{t 1}$ and $p_{t 2}$ around the thrust axis $\hat{n}$.

interpretation due to the entanglement of the involved fragmentation functions in the cross section dependence unlike the simple product of two fragmentation functions present in equation 2.1 in the former reference frame.

The analysis is based on the study of pairs of charged hadrons (C). Unlike sign pairs (U) and like sign pairs (L) are considered separately. These subsamples have indeed a different mix of favoured and disfavoured terms, where favoured means that the fragmenting quark matches one of the valence quark of the produced hadron, for example a $u$ quark fragmenting into a $\pi^{+}$. The BABAR collaboration has extended its analysis published in 2014 measuring Collins asymmetries for pion pairs [2] with a new analysis published at the end of 2015, where the hadrons can be either pions or kaons [3]. It is the first such study involving kaons, thus giving information on the fragmentation of the strange quark. Both $B A B A R$ analyses are based on the total data sample of $468 \mathrm{fb}^{-1}$ taken around $10.6 \mathrm{GeV}$ centre of mass energy.

The analysis needs events with inclusive pairs of charged hadrons in opposite jets. Hadronic events are selected by requiring strictly more than two tracks in the event. The two jets topology is enhanced by requiring the thrust value to be greater than 0.8 , which removes most of the $b \bar{b}$ background. A cut on the event visible energy removes most of the $\tau$ pair background. Tracks are identified as pions or kaons through ionization and Cherenkov radiation measurements. Tracks whose fractional energy lies between 0.15 and 0.9 are considered. This range is divided into four bins in the analysis.

As illustrated by the left plot of figure 2 in the case of kaon pairs in the thrust reference frame, the yields present a cosine dependence. In fact there are other effects, such as the detector acceptance, which may be responsible for a cosine dependence. The Collins effect manifests itself by the difference between the distributions for unlike sign pairs with like sign pairs or with all charged pairs. To remove most of the other effects, double ratios are calculated. They are shown on the right plot of figure 2, for unlike sign over like sign pairs (U/L) and for unlike sign over charged pairs (U/C). The modulation as the function of the angle in these double ratios is mostly due to the Collins effect. Its amplitude is obtained by a fit on the data samples. 


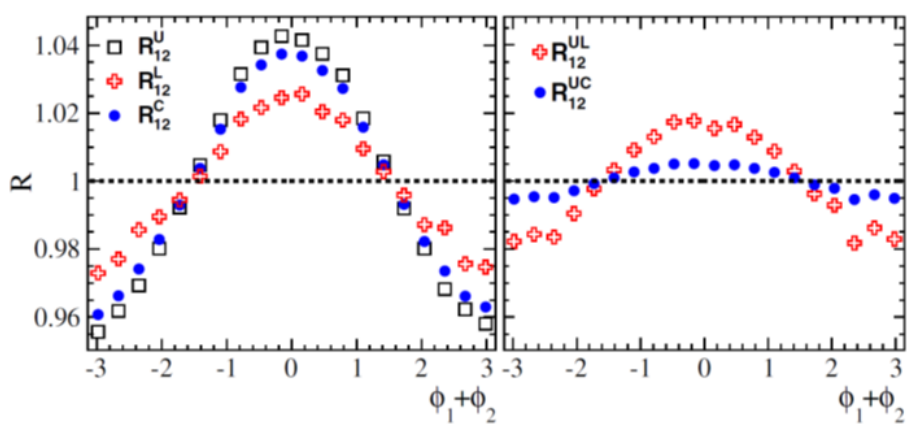

Figure 2: Distributions in the thrust reference frame as a function of the sum of azimuthal angles of (left) normalized yields for (white quares) unlike sign, (red crosses) like sign, and (blue circles) any charge combination of $K K$ pairs and (right) their double ratios, (red crosses) unlike sign over like sign and (blue circles) unlike sign over any charge combination.

In order to extract the asymmetry on $u d s$ events from the measured raw asymmetry simultaneously for $\pi \pi, K \pi$, and $K K$ pairs, we have to correct for both kaon-pion misidentification and backgrounds. The misidentification correction factors are obtained from Monte Carlo. They are smaller than $10 \%$. The main background is from charm. It is about $30 \%$ on average and is estimated on a control sample enhanced with $D^{*}$.

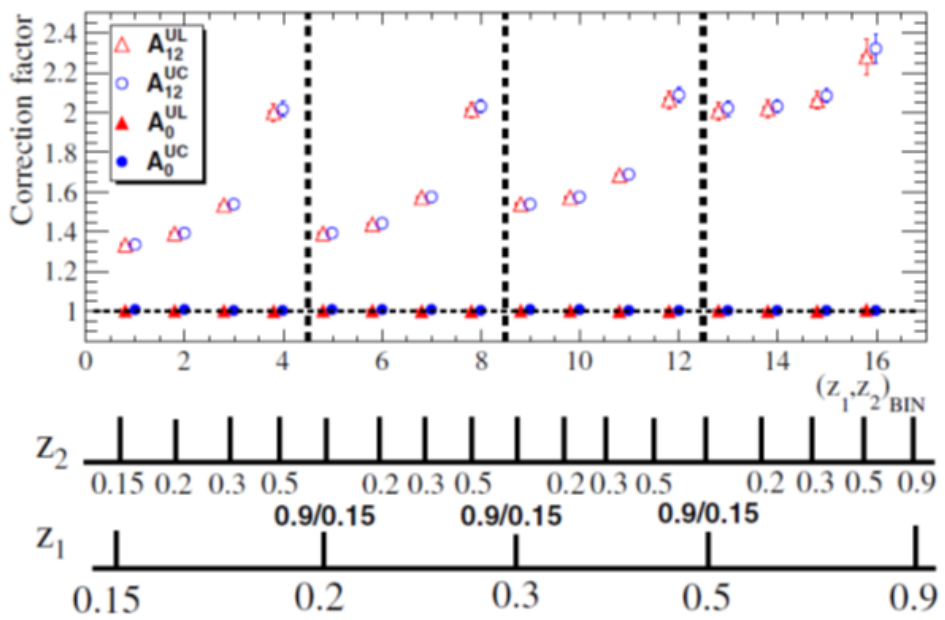

Figure 3: Correction factors for the dilution of the asymmetry due to the difference between the thrust axis and the $q \bar{q}$ direction. They are given by the open (full) markers, red triangles for the U/L and blue circles for the U/C double ratios, in the thrust (other hadron) frame.

Various systematic effects are taken into account. A major one is the dilution of the asymmetry due to the difference between the thrust axis and the $q \bar{q}$ direction. It is relevant only in the thrust reference frame. The correction factors, shown in figure 3 , for all bins in the fractional energies $z_{1}$ and $z_{2}$ of the two hadrons, are determined from Monte Carlo and applied to all data samples. They range from 1.3 to 2.3. Overall, in this analysis, the total relative systematic errors does not exceed $10 \%$. 


\section{Results}

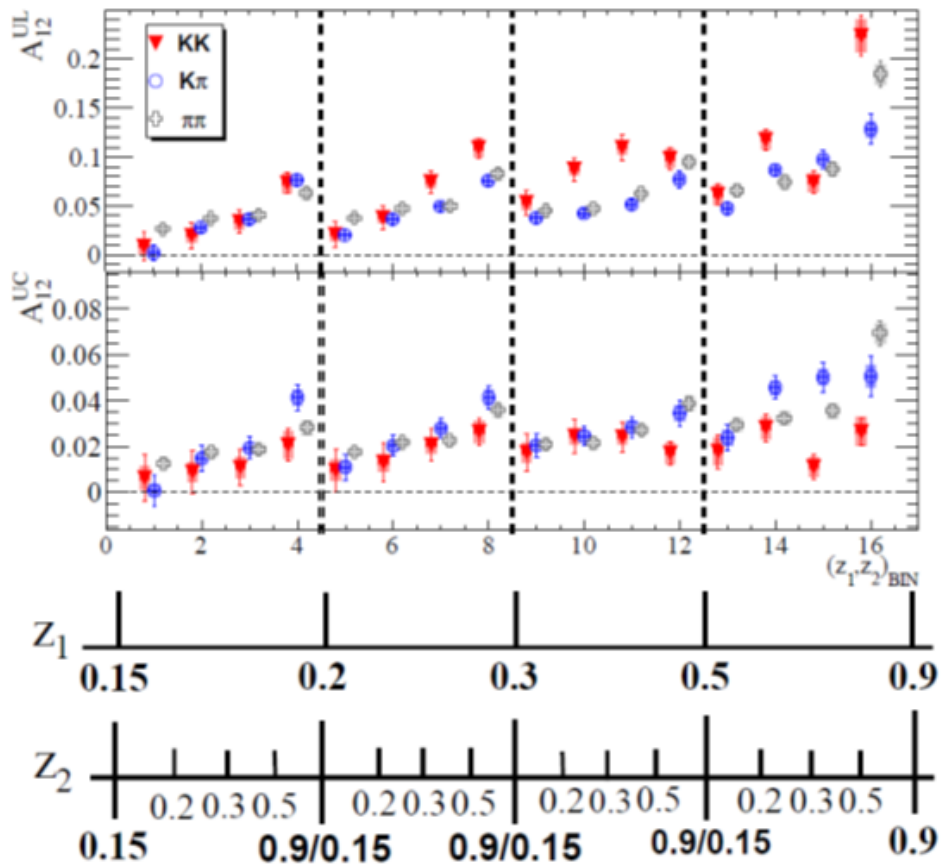

Figure 4: Comparison of (top) $\mathrm{U} / \mathrm{L}$ and (bottom) U/C Collins asymmetries in the thrust reference frame for (red triangles) $K K$, (blue circles) $K \pi$, and (white cross) $\pi \pi$ pairs.

Figure 4 presents the results of the Collins asymmetries in the thust reference frame, as a function of $z_{1}$ and inside each bin of $z_{1}$ as a function of $z_{2}$. A significant asymmetry, which is increasing as a function of the fractional energies, is observed. It is consistent with the expected symmetry between $z_{1}$ and $z_{2}$. The results for $\pi \pi$ are in agreement with previous results from Belle [4] and BABAR [2]. Results for $K \pi$ and $K K$ pairs are obtained for the first time. The asymmetries are of the same magnitude in all channels. The unlike sign to like sign pair asymmetry tends to be higher for kaon pairs at high fractional energy, thus giving an indication on the magnitude of the favored Collins fragmentation function for the strange quark.

The same trends are observed in the second hadron reference frame, as shown in figure 5. In particular, there is again in all modes a significant asymmetry, increasing as a function of $z_{1}$ and $z_{2}$.

\section{Conclusion}

In summary, BABAR has measured Collins asymmetries for charged hadron pairs in two jet events from $e^{+} e^{-}$annihilations in two reference frames. The Collins asymmetries are increasing with fractional energy, in agreement with theoretical expectations. The results for pion pairs agree with previous ones. First measurements have been produced for pairs involving kaons, which give information on the fragmentation of the strange quark. 


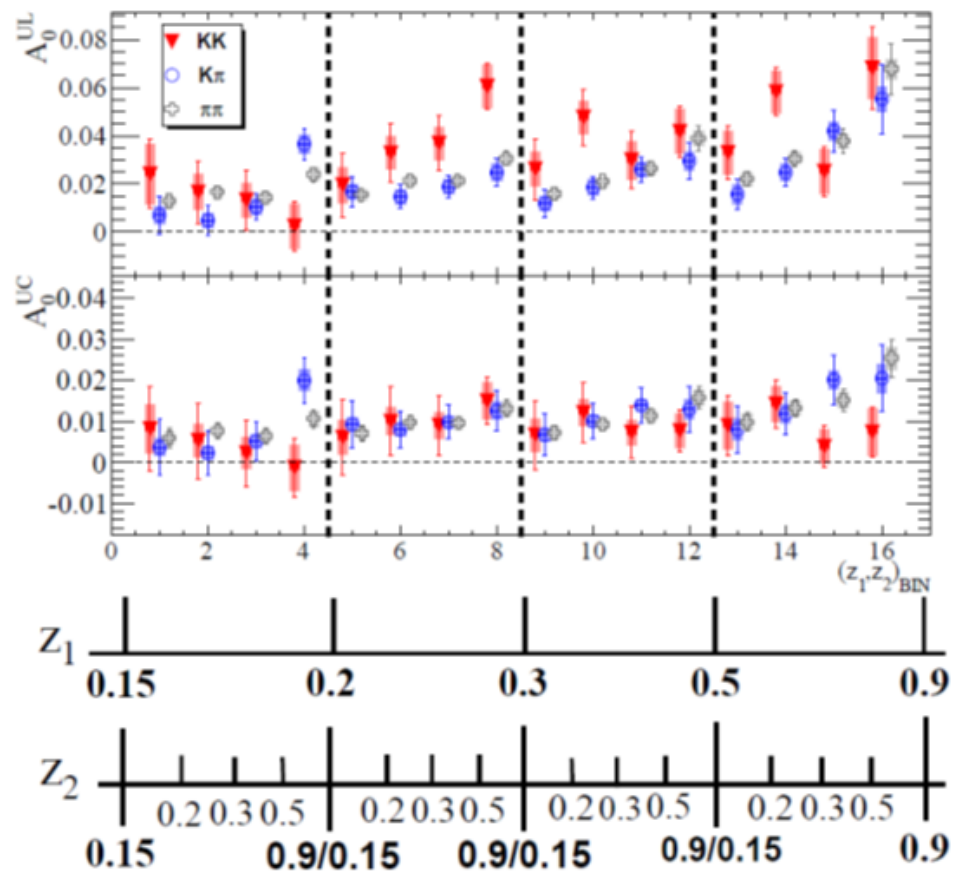

Figure 5: Comparison of (top) U/L and (bottom) U/C Collins asymmetries in the other hadron reference frame for (red triangles) $K K$, (blue circles) $K \pi$, and (white cross) $\pi \pi$ pairs.

\section{References}

[1] J.C. Collins, Fragmentation of transversely polarized quarks probed in transverse momentum distributions, Nucl. Phys. B 396, 161 (1993).

[2] J.P. Lees et al. (BABAR collaboration), Measurement of Collins asymmetries in inclusive production of charged pion pairs in $e^{+} e^{-}$annihilation at BABAR, Phys. Rev. D 90, 052003 (2014).

[3] J.P. Lees et al. (BABAR collaboration), Collins asymmetries in inclusive charged $K K$ and $K \pi$ pairs produced in $e^{+} e^{-}$annihilation, Phys. Rev. D 92, 111101 (2015).

[4] R. Seidl et al. (Belle collaboration), Measurement of azimuthal asymmetries in inclusive production of hadron pairs in $e^{+} e^{-}$annihilation at $\sqrt{s}=10.58 \mathrm{GeV}$, Phys. Rev. D 78, 032011 (2008) and Phys. Rev. D 86, 039905(E) (2012). 